\title{
DEM GENERATION BY MEANS OF NEW DIGITAL AERIAL CAMERAS
}

\author{
J. Höhle
}

Dept. of Planning, Aalborg University, Fibigerstraede 11, DK-9220 Aalborg, Denmark, jh@land.aau.dk

Commission I, WG 2

KEY WORDS: Acquisition, Sensor, CCD, Imagery, Impact Analysis, Accuracy, DEM/DTM

\begin{abstract}
:
Based on practical experiences with the first generation of digital cameras the impact of four new digital aerial cameras on the DEM generation is estimated. The cameras are evaluated with respect to elevation accuracy, area coverage and image quality. In the derived formulae for the absolute accuracy of elevations a camera factor is applied, which summarizes several features in one number. The produced graphs show the potential of the new cameras regarding the relative and absolute accuracy of automatically derived elevations. For better absolute elevation accuracy the increase of the base/height ratio and of the resolving power of lenses are more important than the reduction of the pixel size. Improvement of the elevation accuracy by means of multi-ray photogrammetry is discussed.
\end{abstract}

\section{INTRODUCTION}

10 years use of digital aerial cameras has nearly removed filmbased aerial cameras. The production of orthoimages and DEMs became completely digital, to a large extent automatic and with short response time. These are the major reasons for the success. Regarding the obtainable elevation accuracy with the first generation of the large format frame cameras it was proven in recent tests that they did not perform much better than analogue cameras (Haala et al., 2010). The format is in reality only a fraction of the format of the analogue camera and the base/height ratio is much lower. Several charge-couple devices (CCD) have to be stitched together in order to obtain a larger format. The pixel size of the applied CCD could be reduced in new versions of digital cameras. Small pixel sizes require efficient electronics and storage devices to read out the huge amount of data in a short time. Also lenses with a higher resolving power are necessary to match the small pixel in order to take advantage of high resolution CCDs. Improvement of the economy in aerial photography has been another wish from the camera users. Three camera manufacturers have announced new large format frame cameras, which represent a second generation. Their potential with regard to the elevation accuracy is to the interest of the mapping industry. The development in aerial photogrammetric cameras of one manufacturer can be read from Figure 1. The depicted three cameras represent three generation of cameras: The analogue camera (RMK Top15), the first generation of digital large format frame camera (DMC) and the latest design (DMCII-250). The image area of digital cameras is considerably smaller than the $23 \mathrm{~cm} \times 23 \mathrm{~cm}$ format of film-based aerial cameras. The field of view (FOV) of the selected lenses is also narrower. The panchromatic image of the DMCII-250 camera is produced by a single lens and a single CCD only. Its 250 Megapixel CCD of DALSA has a very small pixel of $5.6 \mu \mathrm{m} \times 5.6 \mu \mathrm{m}$ (Intergraph, 2010). In order to obtain imagery of the same ground sampling distance (GSD) the flying altitude for the DMCII- 250 has to be two times higher than the altitude when using the DMC. The area covered by one image on the ground is, however, 2.4 times larger than the area covered by the DMC. Beside the mentioned camera three other cameras became recently available: The UC-Xp WA and UC Eagle of Microsoft (Microsoft, 2011) and IGI-235 of IGI (IGI,
2010). Their potential regarding DEM generation will be discussed. It is the objective of this contribution to analyze the influencing factors and to predict the results regarding the elevation accuracy at DEM generation with the four new cameras.
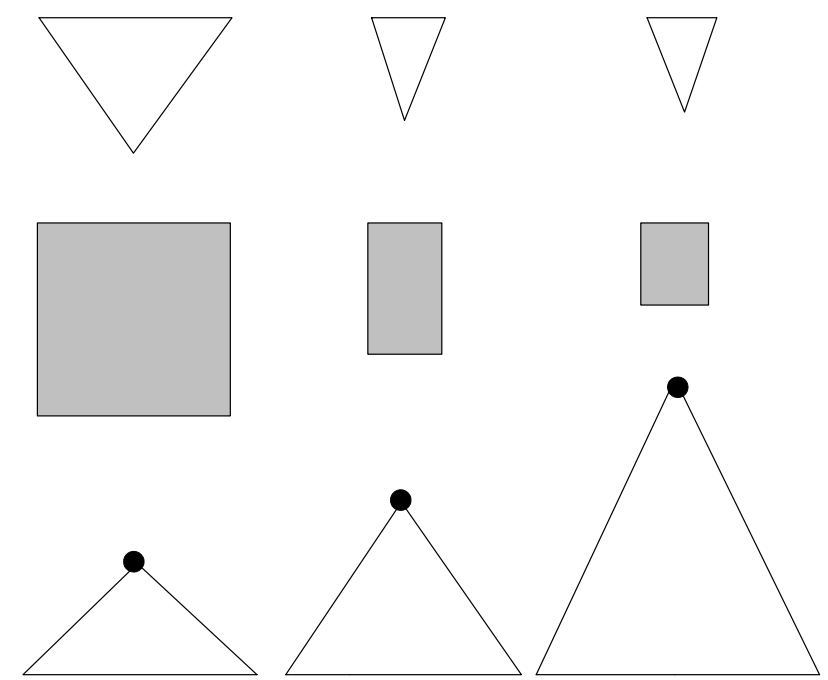

Figure 1. Characteristics of aerial cameras of Intergraph. The symbols mean: From left to right: Film-based wide angle camera, digital cameras DMC and DMCII-250. From top to bottom: FOV in the direction of flight, format, flying height for the same GSD, and FOV across the direction of flight.

\section{INNOVATIONS}

The innovations in the four mentioned cameras first of all concern the number of pixels per image. It reaches now up to 260 million pixels (Mpixel) in the latest design (UC Eagle). The side of the squared pixel is as small as $5.2 \mu \mathrm{m}$. The most recent camera of Intergraph uses one large CCD only which avoids the fusion of several images into one image of a larger format. The image size in flight direction of the DMCII-250 has now 14656 pixels corresponding to $82.07 \mathrm{~mm}$. It is, however, only $36 \%$ of 
the film-based camera. The term "large frame" is related to the number of pixels and not to the physical size of the frame. The Table 1 contains all four new cameras with their features which have influence on the geometric accuracy.

The camera factor $\left(\mathrm{K}_{\mathrm{cam}}\right)$ combines several features in one number. It is derived by:

$$
K_{\text {cam }}=\frac{c}{b^{\prime} \cdot p e l^{\prime}}
$$

where $\mathrm{c}=$ camera constant $b^{\prime}=$ image base at forward overlap of $p=60 \%$ pel'=pixel size.

\begin{tabular}{|c|c|c|c|c|}
\hline Features & IGI 235 & UC-Xp WA & UC Eagle & DMCII 250 \\
\hline pixel size $[\mu \mathrm{m}]$ & 6 & 6 & 5.2 & 5.6 \\
\hline camera constant $[\mathrm{mm}]$ & 80 & 70 & 80 & 112 \\
\hline $\begin{array}{r}\text { image size (in flight direction) }[\mathrm{pel}] \\
{[\mathrm{mm}]}\end{array}$ & $\begin{array}{c}12750 \\
76.50\end{array}$ & $\begin{array}{c}11310 \\
67.86\end{array}$ & $\begin{array}{c}13080 \\
68.02\end{array}$ & $\begin{array}{l}14656 \\
82.07\end{array}$ \\
\hline GSD at flying height of $\mathrm{h}=1000 \mathrm{~m}[\mathrm{~cm}]$ & 7.5 & 8.6 & 6.5 & 5.0 \\
\hline base/height ratio at $\mathrm{p}=60 \%$ & 0.38 & 0.39 & 0.34 & 0.29 \\
\hline $\mathrm{K}$-factor $\left[\mu \mathrm{m}^{-1}\right]$ & 0.436 & 0.430 & 0.565 & 0.609 \\
\hline
\end{tabular}

Table 1. Features of four new digital aerial cameras

If the images have the standard forward overlap of $p=60 \%$, the image base is $b^{\prime}=0.4 \cdot \mathrm{s}_{\text {fld }}$, where $\mathrm{s}_{\text {fld }}=$ image size in flight direction.

From Table 1 it can be noticed that the camera factor $\left(\mathrm{K}_{\mathrm{cam}}\right)$ is lowest for the IGI-235 camera and highest for the DMC-250 camera. The older digital cameras have even smaller factors: 0.412 (UC-D) and 0.269 (DMC). High elevation accuracy is achieved with low camera factors. Other camera features like frame rate and image quality have also influence on the generation of DEM and will also be dealt with in the following.

\subsection{Geometric accuracy}

The performance of digital aerial cameras has recently been investigated by several research groups (Jacobsen, 2011), (Haala et al., 2010), (Spreckels et al., 2010), (Höhle, 2009a\&b). Besides the camera also the flight parameters (altitude, side lap) and the processing tools influence the accuracy of derived elevations. Furthermore, the terrain type, the density, and the definition of the points have an effect. The assessment of the accuracy needs checkpoints of superior accuracy. The applied accuracy measures should not be affected by blunders.

In the investigation of the DMCII-140, which uses one 140 Mpixel CCD with a $7.2 \mu \mathrm{m} \times 7.2 \mu \mathrm{m}$ large pixel, a vertical accuracy of $\mathrm{RMSE}_{\mathrm{h}}=0.7 \cdot \mathrm{GSD}$ has been achieved for well defined checkpoints using semi-automated measurements (Jacobsen, 2011). The imagery used $65 \%$ forward overlap and $65 \%$ side overlap and the determination of the check- and control points could in average use seven images. The object points have been determined within an aerotriangulation. The achieved high accuracy is, therefore, not a result of a DEM generation.

In tests of a project group of the German society of photogrammetry, remote sensing and geoinformation (DGPF) it was found that the generation of very dense DEMs by means of the DMC images with GSDs of $8 \mathrm{~cm}$ and $20 \mathrm{~cm}$ could be done with $\mathrm{RMSE}_{\mathrm{h}}=0.4 \cdot \mathrm{GSD}$ and $\mathrm{RMSE}_{\mathrm{h}}=0.8 \cdot \mathrm{GSD}$ respectively (Haala et al., 2010). The checkpoints were well defined and with good contrast to the surroundings, and the derived DEM had a very narrow spacing of the grid points. A few blunders were eliminated by a threshold ( $3 \cdot$ RMSE).

High elevation accuracies of $\sigma_{\mathrm{h}}=0.45 \cdot \mathrm{GSD}$ were obtained when well defined points were manually measured in DMC images by an experienced operator (Spreckels et al., 2010).

Other investigations were carried out in (Höhle, 2009a) and (Höhle, 2009b) using UltraCam-D and DMC images. The results from these investigations will be used for estimating the impact of the four new digital aerial cameras.

In order to estimate the results with the new cameras the factors influencing the accuracy have to be known. In general, the estimates of the absolute elevation accuracy can be calculated after the formula (2).

$\sigma_{h}=\frac{h}{b} \cdot \sigma_{p x^{\prime}} \cdot m_{b}$

where $\sigma_{\mathrm{h}}=$ elevation accuracy, $\mathrm{h}=$ (mean) flying height above ground, $\mathrm{b}=$ basis, $\sigma_{\mathrm{px}}=$ parallax accuracy related to the image, $\mathrm{m}_{\mathrm{b}}=$ image scale figure.

The formula can be 'modernized' with regard to digital cameras:

$\sigma_{h}=\frac{c}{b^{\prime}} \cdot \frac{\sigma_{p x^{\prime}}}{p e l^{\prime}} \cdot G S D$

When applying the factor $\mathrm{K}_{\mathrm{cam}}$ the absolute elevation accuracy is obtained by means of formula (4):

$\sigma_{h}=K_{c a m} \cdot \sigma_{p x^{\prime}} \cdot G S D$

where

$G S D=p^{\prime} \cdot m_{b}=p e l \cdot \frac{h}{c}$.

The relative elevation accuracy is then derived by formula (5). 


$$
\frac{\sigma_{h}}{h}=\frac{\sigma_{p x^{\prime}}}{b^{\prime}}
$$

The image base (b') may be determined from the number of pixels in the direction of flight $\left(\mathrm{s}^{\prime}{ }_{\text {fld }}\right)$ assuming $60 \%$ forward overlap. The relative accuracy of parallaxes $\left(\sigma_{\mathrm{px}}, / \mathrm{pel}\right.$ ') can empirically be derived by means of equation (6).

$$
\frac{\sigma_{p x^{\prime}}}{p e l^{\prime}}=\frac{b^{\prime}}{c} \cdot \frac{\sigma_{h}}{G S D}
$$

In (Höhle, 2009b) the relative accuracy has been derived for the DMC camera (cf. Table 2). The required value for the absolute accuracy $\left(\sigma_{h}\right)$ has been found from comparison with accurate reference points.

\begin{tabular}{|c|c|c|c|c|c|c|}
\hline $\begin{array}{c}\mathrm{b} / \mathrm{c} \\
\mathrm{b} / \mathrm{h}\end{array}$ & $\begin{array}{c}\text { GSD } \\
{[\mathrm{cm}]}\end{array}$ & $\begin{array}{c}\text { spacing } \\
{[\mathrm{m}]}\end{array}$ & $\begin{array}{c}\sigma_{h} \\
{[\mathrm{~cm}]}\end{array}$ & $\frac{\sigma_{h}}{G S D}$ & $\begin{array}{c}\sigma_{\mathrm{px}} \\
{[\mu \mathrm{m}]}\end{array}$ & $\frac{\sigma_{p x^{\prime}}}{p e l^{\prime}}$ \\
\hline 0.31 & 20 & 3.0 & 17.0 & 0.85 & 3.2 & 0.26 \\
\hline 0.31 & 10 & 1.6 & 15.0 & 1.50 & 5.5 & 0.46 \\
\hline
\end{tabular}

Table 2. Results of automatic DTM derivation in open terrain using the DMC camera $\left(\mathrm{c}=120 \mathrm{~mm}\right.$, pel' $=12 \mu \mathrm{m}, \mathrm{s}^{\prime}{ }_{\text {fld }}=7680$ pixel, $\mathrm{b} / \mathrm{h}=0.31$ at $\mathrm{p}=60 \%)$, standard overlap $(\mathrm{p}=60 \%, \mathrm{q}=20 \%)$ and processing with Match-T

The mean of the relative parallax accuracy is 0.36 which corresponds to an absolute parallax accuracy of $\sigma_{\mathrm{px}}=4.3 \mu \mathrm{m}$. This value is independent from the used overlap. It should be a constant value for the DMC camera provided that the orientation of the images is error-free and the terrain is not covered with vegetation or buildings.

Other cameras may have other values. For example, in (Höhle 2009a) the parallax accuracy has been determined for the UC-D camera $(\mathrm{c}=101 \mathrm{~mm}$, pel' $=9 \mu \mathrm{m}, \mathrm{b} / \mathrm{h}=0.27$ at $\mathrm{p}=60 \%$,

$\mathrm{s}_{\mathrm{fld}}=7500$ pixel) under similar conditions $(\mathrm{GSD}=6 \mathrm{~cm}, \mathrm{p}=60 \%$, $\mathrm{q}=20 \%$, processing with Match-T) and the parallax accuracy has been $\sigma_{\mathrm{px}}=6.0 \mu \mathrm{m}$ or 0.67 . The average from all three tests is $\left(\sigma_{\mathrm{px}}, \mathrm{pel}^{\prime}\right)_{\mathrm{average}}=0.46 \approx 0.5$.

The results obtained in the above mentioned tests cannot directly be compared with results of tests using standard overlap and single models for the automated generation of elevations of natural terrain points. In the following accuracy estimation for the four new cameras it is assumed that the relative parallax accuracy is $\sigma_{\mathrm{px}}, / \mathrm{pel}{ }^{\prime}=0.5$ as well. The figure 2 depicts the relative elevation accuracy and Figure 3 the absolute accuracy of the elevations which the four new cameras may achieve.

From Figure 2 it can be read that the relative accuracy $\left(\sigma_{\mathrm{h}} / \mathrm{h}\right)$ will improve at all of the new cameras. However, the increase in the number of pixels in the direction of flight does not mean that also the absolute accuracy improves (cf. Figure 3). Both the base/height ratio and the image scale have influence on the absolute elevation accuracy. The absolute elevation accuracy with the DMCII-250 will not be better than the results with the DMC. In order to obtain a vertical accuracy of $\sigma_{\mathrm{h}}=10 \mathrm{~cm}$, the DMCII-250 images have to be taken from an altitude $\mathrm{h}=1180 \mathrm{~m}$ (corresponding to $\mathrm{GSD}=5.9 \mathrm{~cm}$ ).

When mass points for the purpose of terrain modelling are automatically determined the terrain type will have some influence. Terrain areas with buildings and vegetation have to be filtered and arising gaps have to be closed by interpolation. Errors will occur in these processes and the obtainable accuracy is then less than in open terrain. In (Höhle 2010) the DTM accuracy in built-up areas has been assessed with $\sigma_{\mathrm{h}}=16.5 \mathrm{~cm}$ or $0.165 \% \cdot \mathrm{h}$. The applied DMC imagery had standard overlaps $(\mathrm{p}=60 \%$ $\mathrm{q}=20 \%$ ) and $\mathrm{GSD}$ of $10 \mathrm{~cm}$.

\subsection{Coverage in object space}

Due to a newly designed lens the format of the CCD in the DMCII-250 is completely used for imaging. The ground coverage at $\mathrm{GSD}=10 \mathrm{~cm}$ is $1722 \mathrm{~m} \times 1466 \mathrm{~m}$ or $2.52 \mathrm{~km}^{2}$. The UC Eagle and the IGI-235 have about the same footprint (cf. Table 3).

A large 'footprint' is an economic factor. The flying altitude for the DMCII-250 has to be higher, which may reduce contrast and colour balancing in the images.

\subsection{Increase of overlap and combined use of imagery}

The accuracy can be improved when the elevations are determined from several images. This multi-ray approach is achieved by higher overlaps. The increase of the forward overlap to $80 \%$ is achieved almost without additional costs. The small frame rate of the new cameras, e.g. 1.7 seconds at the DMCII-250, makes a ground resolution of $\mathrm{GSD}=5 \mathrm{~cm}$ possible at ground speeds of the aircraft up to $310 \mathrm{~km} / \mathrm{h}$. Figure 4 depicts an image together with the centres of four overlapping images and Table 4 shows base/height ratios for various combinations of image pairs. The elevations are derived from image pairs and have to be merged or the intersection of points is carried out simultaneously from several images. Special software for the processing is then necessary and also available from different vendors, e.g. (Erdas, 2010), (Inpho, 2011). Using several photogrammetric models and merging the derived DEMs will reduce the number of blunders and improve the accuracy. The reduction of the standard deviation may be estimated by:

$\sigma_{h_{-} \text {average }}=\frac{1}{\sqrt{n_{\bmod }}} \cdot \sigma_{h}$

where $\quad n=$ number of models.

The accuracy is also improved by means of higher side-overlap, e.g. of $\mathrm{q}=60 \%$. This approach increases the cost of flying. Generally, a large base line leads to differences in the perspective distortion, which may create blunders at large scale imagery. In contrast, a small base line will improve the matching accuracy, but the elevation accuracy will suffer due to the unfavourable base/height ratio. The error propagation in multi-ray photogrammetry for DEM generation needs some further investigation and practical experience. The current tests of a benchmarking may give some answers in this respect (EuroSDR, 2011).

Of advantage is also the approach with two flying heights. It may be practiced in projects where imagery is taken for built-up areas with $\mathrm{GSD}=10 \mathrm{~cm}$ and for the whole territory with $\mathrm{GSD}=20 \mathrm{~cm}$ and applied for orthoimage generation and stereo compilation. When using such imagery for DEM generation a $17 \%$ improvement in the standard deviation in the open land and $42 \%$ in the built-up areas was obtained when merging several DEMs (Höhle, 2009b). 


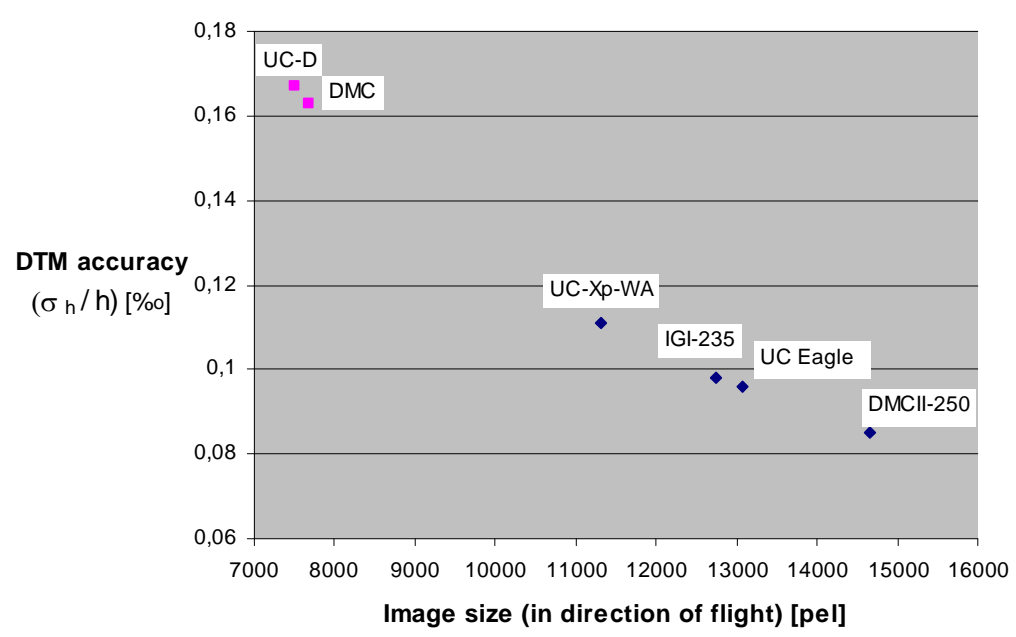

Figure 2. Relative elevation accuracy of digital aerial cameras

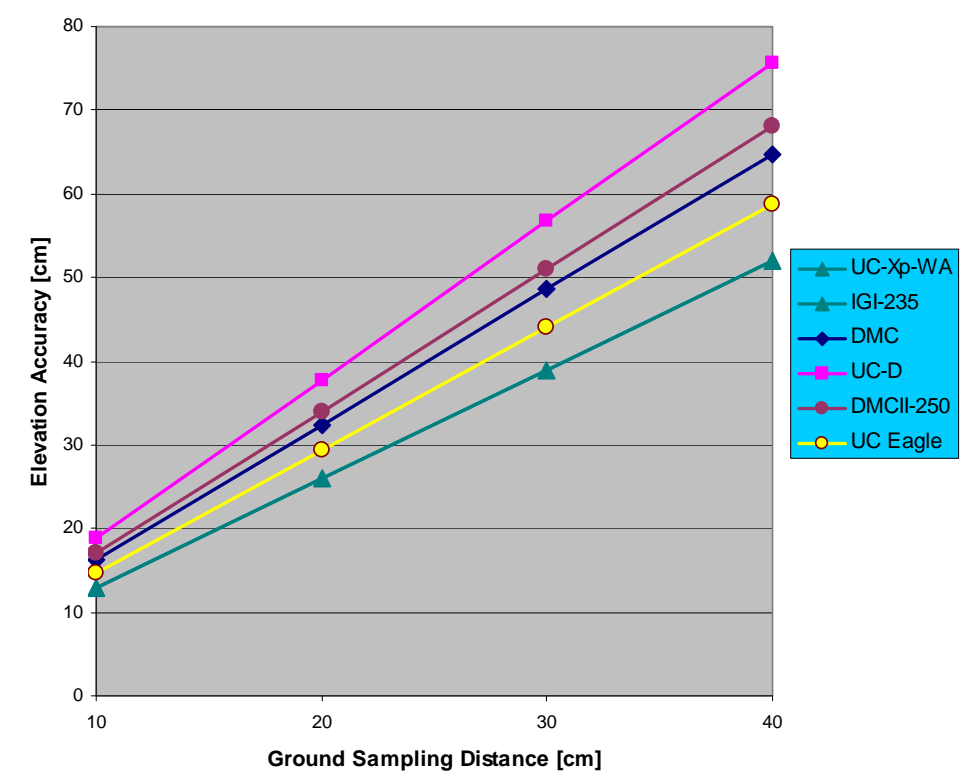

Figure 3. Absolute elevation accuracy for digital cameras as function of GSD

\begin{tabular}{|c|c|c|c|c|}
\hline Features & IGI 235 & UC-Xp WA & DMCII 250 & UC Eagle \\
\hline flying height at $\mathrm{GSD}=10 \mathrm{~cm}$ & 1333 & 1167 & 2000 & 1538 \\
\hline \begin{tabular}{|cc} 
image size (across flight direction) [pel] \\
& {$[\mathrm{mm}]$} \\
at $\mathrm{GSD}=10 \mathrm{~cm}$ & {$[\mathrm{~m}]$} \\
\end{tabular} & $\begin{array}{l}18500 \\
111.0 \\
1850 \\
\end{array}$ & $\begin{array}{c}17310 \\
103.9 \\
1731 \\
\end{array}$ & $\begin{array}{c}17216 \\
96.4 \\
1722 \\
\end{array}$ & $\begin{array}{l}20010 \\
104.1 \\
2001 \\
\end{array}$ \\
\hline \begin{tabular}{|ll} 
Image size (in flight direction) & {$[\mathrm{pel}]$} \\
& {$[\mathrm{mm}]$} \\
& {$[\mathrm{m}]$} \\
\end{tabular} & $\begin{array}{c}12750 \\
76.50 \\
1275 \\
\end{array}$ & $\begin{array}{c}11310 \\
67.86 \\
1131 \\
\end{array}$ & $\begin{array}{c}14656 \\
82.07 \\
1466 \\
\end{array}$ & $\begin{array}{c}13080 \\
68.02 \\
1308 \\
\end{array}$ \\
\hline ground coverage at $\mathrm{GSD}=10 \mathrm{~cm} \quad\left[\mathrm{~km}^{2}\right]$ & 2.36 & 1.96 & 2.52 & 2.62 \\
\hline relative coverage & 90 & 75 & 96 & 100 \\
\hline
\end{tabular}

Table 3. Ground coverage of the four new digital cameras 


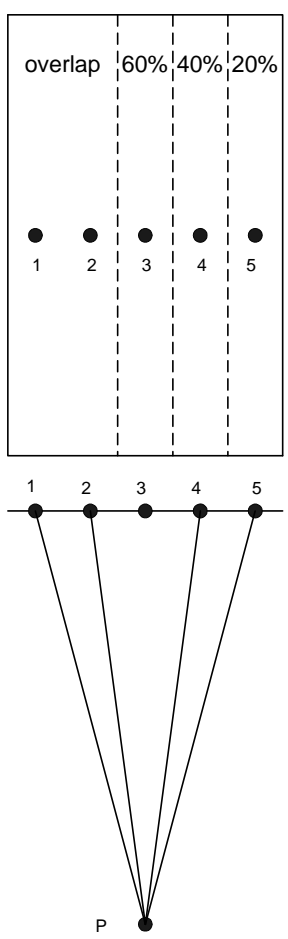

Figure 4. Multiple image pairs may be used to determine the elevation of an object point (P). For image pairs with less overlap the base/height ratio is increased and the accuracy of the derived elevations is improved.

\begin{tabular}{|c|c|c|}
\hline Image pair & overlap & base/height ratio \\
\hline 1,5 & $20 \%$ & $1 / 1.9$ \\
\hline 1,4 & $40 \%$ & $1 / 2.5$ \\
\hline 2,5 & $40 \%$ & $1 / 2.5$ \\
\hline 2,4 & $60 \%$ & $1 / 3.7$ \\
\hline $2,3 / 3,4$ & $80 \%$ & $1 / 7.5$ \\
\hline
\end{tabular}

Table 4. Image pair combinations and the base/height ratios of the digital large format camera UltraCam-D.

\subsection{Image quality}

Important for good results in geometry is also the quality of the produced image. Regarding the panchromatic camera of the DMCII-250, the resolving power of the new Carl Zeiss lens has to match the size of the small pixel. A filter removes light beyond the wavelength of $\lambda=710 \mathrm{~nm}$. Colour and colour infra-red images are produced by means of pan-sharpening from four multi-spectral cameras of lower resolution and smaller camera constant. The PAN/colour ratio is 1:3.2. The forward image motion due to speed of the aircraft is compensated in all four cameras by time-delayed integration (TDI). The DMCII-250 camera has a radiometric resolution of 14bit (or 16384 intensity values) for each pixel. All this is an important condition for high parallax accuracy. Details of the radiometric postprocessing are dealt with e.g. in (Honkavaara et al., 2009). The other three new cameras have similar features regarding the radiometric quality. The IGI-235 (also called Quattro DigiCAM) has interchangeable lenses with focal lengths of $f=80,100$, and $150 \mathrm{~mm}$. The UC Eagle can also be used with a $210 \mathrm{~mm}$ lens. DEM generation is best done with lenses of small focal length. It is important to understand that a small pixel will not necessarily result in high resolution of the image. The resolving power of the lens has to be equally high. A CCD with pixels of $5.6 \mu \mathrm{m} \cdot 5.6 \mu \mathrm{m}$ requires a lens with a resolution of $\mathrm{R}=1000 /(2 \cdot 5.6 \mu \mathrm{m})=89$ line pairs per $\mathrm{mm}(\mathrm{lp} / \mathrm{mm})$. The resolution of the lens decreases from the middle of the lens to the corners. Furthermore, diffraction will occur when high f-numbers have to be used for correct exposures. The limitation of resolution of a lens due to diffraction is computed after

$$
\mathrm{x}=1.2 \cdot \lambda \cdot \mathrm{b}
$$

where $\mathrm{x}=$ smallest distance, which can be resolved, $\lambda=$ wavelength of the used light, and $b=f$-number.

(Schwidefsky, 1976, p.48).

Assuming $\lambda=0.575 \mu \mathrm{m}$ and $\mathrm{b}=16$ then $\mathrm{x}=11 \mu \mathrm{m}$.

The resolution of the lens at this f-number $(b=16)$ is limited to $91 \mathrm{lp} / \mathrm{mm}$ due to diffraction. This means that exposures with $\mathrm{f}-$ number $>16$ cannot take advantage of the resolution given by a pixel size of $(5.5 \mu \mathrm{m})^{2}$.

\section{DISCUSSION}

The recent announcement of new digital aerial cameras has started this investigation to estimate their impact on automated DEM acquisition. All four cameras have a much larger number of pixels per final output image than the cameras UC-D and DMC. It is remarkable that the 250 Megapixels of the panchromatic camera of the DMCII are achieved with one CCD array and one lens. This fact will improve the geometric accuracy because errors in the fusion of several CCDs are avoided and only one perspective centre exists. The pixel sizes in all cameras are now very small, which results in higher flying altitudes. Then the relative accuracy is better, but important for the user is the absolute accuracy for a given GSD.

The empirical tests with the first generation cameras (DMC and UC-D) revealed parallax accuracies of about 0.5 pixels in average. Applying this value for all of the new cameras the absolute accuracy will then be higher for the large-format cameras (IGI235 and UC-Xp WA) due to their bigger base/height ratio (0.38 and 0.39 respectively). The one-chip camera (DMCII-250) comes close to the results of the large-format camera DMC. It is also advantageous that the elevations can be determined by several images due to the short frame rate in the new cameras. This multi-ray photogrammetry requires higher side overlap and special software packages. Practical experience is still necessary in order to use this approach with its full potential.

An alternative solution is the determination of DEMs from images taken at different altitudes and using the standard forward overlap ( $\mathrm{p}=60 \%$ ) and merging the different DEMs. The completeness, reliability and the accuracy of the DEMs can be improved by means of this approach.

The economy of DEM generation will be improved with the four new cameras. The swath width is increased either due to a larger field of view (UC-Xp WA, IGI, 235) or due to a higher flying height (DMCII-250, UC Eagle), which the small pixel or the longer focal length require. The ground resolution, however, will still be high. In order to recognize and measure small objects like manhole covers and drain gratings a high ground resolution is necessary. In the mapping industry is a tendency to use imagery with GSD smaller than $10 \mathrm{~cm}$. 
The high resolution and image quality of the new digital cameras enable very dense point clouds at the automated DEM generation. The terrain can be modelled more accurately. Break lines, buildings and other objects can automatically be extracted more accurately and reliably.

\section{CONCLUSION}

Determination of elevations and DEMs will benefit from using one of the new generations of cameras. The improvements are due to a more favourable base/height ratio at the UC-Xp WA and IGI 235 cameras and at the DMCII-250 where the fusion of several CCDs is avoided. In this case the format of the image becomes nearly a square, which then matches the FOV of the single lens. The UC Eagle with its 260 Mega pixel per final output image sets a new scale regarding the amount of pixels.

Applying a relative parallax accuracy of $\sigma_{\mathrm{px}}{ }^{\prime} / \mathrm{pel},=0.5$ for all four new cameras the achievable accuracy can be estimated and compared. In order to obtain a vertical accuracy of $\sigma_{\mathrm{h}}=10 \mathrm{~cm}$ the images have to be taken with a ground sampling distance of $\mathrm{GSD}=5.9 \mathrm{~cm}$ (DMCII-250), $6.8 \mathrm{~cm}$ (UC Eagle), $7.6 \mathrm{~cm}$ (IGI-235, $\mathrm{c}=80 \mathrm{~mm})$, or $7.8 \mathrm{~cm}$ (UC-Xp WA).

The ground coverage of the UC Eagle is largest (100\%), 96\% of the DMCII- $250,90 \%$ of the IGI-235 camera, and $75 \%$ of the UC-Xp WA camera ( $80 \mathrm{~mm}$ lens) when the same ground resolution is maintained. Due to a short frame rate the multi-ray and multi-model techniques can be applied at all new cameras which will further improve the vertical accuracy. Other applications, e.g. the extraction of break lines or buildings, will benefit from the new cameras too.

Practical tests with the new cameras have to be carried out in order to confirm these theoretical considerations.

With the appearance of the four new aerial cameras the development of digital aerial cameras is not finished. The CCDs may become larger and squared and used in single chip or multi-chip designs. The image quality and other features of the cameras will improve in future as well. All this progress in the cameras and the processing tools will definitely improve the DTM/DSM generation by means of photogrammetry.

\section{REFERENCES}

EuroSDR, 2011. Benchmarking of Image Matching Approaches for DSM Computation,

http://eurosdrbenchmarkofimagematching.ign.fr/ (accessed 15.5.2011)

Grenzdörffer, G., 2010. Medium Format Cameras, EuroSDR Official Publication no. 58, pp. 233-262.

Haala, N., Hastedt, H., Wolf, K., Ressl, C., Baltrusch, S., 2010. Digital Photogrammetric Camera Evaluation - Generation of Digital Elevation Models, PFG 2/2010, pp. 99-115.

Höhle, J., 2009a. DEM Generation Using a Digital Large Format Frame Camera, Photogrammetric Engineering \& Remote Sensing (PE\&RS), Vol. 75, no.1, pp. 87-93.

Höhle 2009b, Updating of the Danish Elevation Model by means of photogrammetric methods, National Survey and Cadastre-Denmark, technical report series number 03, ISBN8792107-25-7, 64 p.,
http://www.kms.dk/NR/rdonlyres/1C10C559-6CC9-452085C5-DE8659CB38A9/0/kmsrep_3.pdf (accessed 23.4.2011).

Höhle, J., 2010. Generation and Application of Digital Elevation Models, dissertation at Aalborg University, 177 p., $\mathrm{http}: / /$ people.plan.aau.dk/ jh/Articles/Hoehle_D_final.pdf (accessed 23.4.2011).

Honkavaara, E., Arbiol, R., Markelin, L., Martinez, L., Cramer, M., Bovet, S., Chandelier, L., Ilves, R., Klonus, S., Marshal, P., Schläpfer, D., Tabor, M., Thom, C., Veje, N., 2009. Digital Airborne Photogrammetry-A New Tool for Quantitative Remote Sensing? - A State-of-the-Art Review On Radiometric Aspects of Digital Photogrammetric Images, Remote Sensing, vol. 1, pp. 577-605.

Sandau, R. (editor), 2005. Digitale Luftbildkamera, Einführung und Grundlagen, Wichmann Verlag, ISBN 3-87907-391-0, $342 \mathrm{p}$.

Spreckels, V. Syrek, L., Schlienkamp, A., 2010. DGPF Project: Evaluation of Digital Photogrammetric Camera Systems Stereoplotting, PFG 02/2010, 14p.

Schwidefsky, K., Ackermann, F., 1976. Photogrammetrie, 7th edition, B.G. Teubner, Stuttgart. ISBN 3-519-13401-2

\section{COMPANY LITERATURE}

Erdas, 2011. LPS 2011, brochure, 16 p., http://www.erdas.com/Libraries/Tech_Docs/LPS_2011_Product _Description.sflb.ashx (accessed 17.5.2011)

IGI, 2010. Quattro DigiCAM-Modular Large Format Aerial Camera, brochure, 2p., http://www.igi.eu/brochures.html, (accessed 26.4.2011).

Inpho, 2011. Match-T DSM, brochure, 2p., http://www.inpho.de/index.php?seite=index matcht\&navigation $=185$ \& root $=165 \& \mathrm{kanal}=\mathrm{html}$ (accessed 13.6.2011)

Intergraph, 2010. DMC® II250 CAMERA SYSTEM, brochure, $2 \mathrm{p}$,

http://www.intergraph.com/assets/plugins/sgicollaterals/downlo ads/DMCII250-CameraSystem_ProductSheet.pdf (accessed 26.4.2011).

Microsoft, 2011, UltraCamXp WA, Technical Specifications, 1p., http://www.microsoft.com/ultracam/enus/UltraCamXpWATechnical.aspx (accessed 13.5.2011)

\section{ACKNOWLEDGEMENTS}

The author thanks the unknown reviewers for their constructive criticism. 